\title{
II Seminario Internacional «El Derecho a la ciudad en América Latina: lo político de las políticas urbanas»
}

Lima, 21 y 22 de agosto de 2014

Jérémy Robert y Cyriaque Hattemer

\section{(2) OpenEdition}

Journals

Edición electrónica

URL: http://journals.openedition.org/bifea/5312

DOI: $10.4000 /$ bifea. 5312

ISSN: 2076-5827

Editor

Institut Français d'Études Andines

Edición impresa

Fecha de publicación: 1 agosto 2014

Paginación: 413-416

ISSN: 0303-7495

\section{Referencia electrónica}

Jérémy Robert y Cyriaque Hattemer, « II Seminario Internacional «El Derecho a la ciudad en América Latina: lo político de las políticas urbanas» », Bulletin de l'Institut français d'études andines [En línea], 43 (2) | 2014, Publicado el 08 agosto 2014, consultado el 10 diciembre 2020. URL : http:// journals.openedition.org/bifea/5312; DOI : https://doi.org/10.4000/bifea.5312

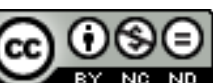

Les contenus du Bulletin de l'Institut français d'études andines sont mis à disposition selon les termes de la licence Creative Commons Attribution - Pas d'Utilisation Commerciale - Pas de Modification 4.0 International. 


\section{SEMINARIO INTERNACIONAL «EL DERECHO A LA CIUDAD EN AMÉRICA LATINA: LO POLÍTICO DE LAS POLÍTICAS URBANAS»1}

Lima, 21 y 22 de agosto de 2014

Los días 21 y 22 de agosto de 2014 se realizó en Lima el II Seminario Internacional impulsado por el Grupo de Trabajo «El Derecho a la Ciudad en América Latina» (GT-DC), del Consejo Latinoamericano de Ciencias Sociales (CLACSO), coordinado por Fernando Carrión y María Cristina Cravino. Ha sido el resultado del esfuerzo de un conjunto de instituciones: acogido por la Universidad Católica del Perú (PUCP), coorganizado por la Facultad de Ciencias sociales, el Departamento de Ciencias Sociales y Centro de Investigación de la Arquitectura y la Ciudad (CIAC) de la misma universidad, el Instituto Francés de Estudios Andinos (IFEA, UMIFRE 17 MAEDI/CNRS USR 3337 - Amérique Latine, Lima, Perú) y la CLACSO, con el apoyo de la Municipalidad Metropolitana de Lima, la Facultad de Ciencias Sociales FLACSO Ecuador, la Universidad Nacional de Ingeniería del Perú (UNI), la Universidad Ricardo Palma, el Centro de Investigación, Documentación y Asesoría Poblacional (CIDAP), el Centro de Estudios Mexicanos y Centroamericanos (CEMCA) y la Organización Internacional para las Migraciones (OIM).

1 La información del evento está disponible en http://seminario.pucp.edu.pe/derecho-ciudad/ y las videoconferencias en http://educast.pucp.edu.pe/video/3893/ii_seminario_continuo_el_derecho a_la_ciudad_en_america_latina_lo_politico_de_las_politicas_urbanas_inauguracion?autoplay = true 
El Grupo de Trabajo «El Derecho a la Ciudad en América Latina» ha sido conformado con el propósito de discutir y reflexionar acerca de los contenidos teóricos y las experiencias desarrolladas en torno a esta temática de gran actualidad en la región de América Latina y el Caribe. El evento realizado en Lima se inscribe en la continuidad de un primer evento realizado en la ciudad de México en febrero de 2014, seguido por dos eventos anuales que se llevarán a cabo en 2015 (en Santiago de Chile y Buenos Aires) y 2016 (lugar por definir).

La llegada del evento en la ciudad de Lima responde al interés de varias instituciones y elementos de coyuntura, en particular la campaña por la alcaldía de Lima. Se inscribe también en una larga tradición de colaboración científica entre el IFEA y América Latina. Se puede recordar un evento similar por la temática y el contexto acerca de los servicios públicos de Lima durante la campaña electoral de 1989 coorganizado por el Centro de Investigación de la Universidad el Pacífico (CIUP) y el IFEA, que dio lugar a la publicación de «Lima en crisis» (Figari Gold \& Ricou, 1991).

Esta segunda edición «El Derecho a la Ciudad en América Latina: Lo político de las políticas urbanas» se enfocó en la dimensión política del Derecho a la Ciudad. Incluye, por un lado, el ensamble del conjunto de los derechos en tanto son interdependientes y, por otro lado, un derecho de expresión de una reivindicación y un proyecto colectivo de ciudad. La propuesta era llevar a cabo una reflexión sobre la posibilidad política de incidir en las políticas, es decir no solamente contemplar los mecanismos de participación, sino analizar la oportunidad de los diferentes grupos sociales de incidir sobre las decisiones y la producción de la ciudad.

Ha sido la oportunidad de discutir un tema no tan trabajado y a veces olvidado de las investigaciones urbanas de la región, subrayando las transformaciones contemporáneas del contexto político de América Latina. Se trata por ejemplo del «giro a la izquierda» de los gobiernos de las principales metrópolis de la región, que merece la atención de las ciencias sociales, la renovación de sus enfoques y reinterrogar sus conceptos como el de gobernanza o ciudadanía².

El Derecho a la Ciudad se planteó entonces como un eje transversal en el debate actual sobre las transformaciones urbanas en la región y fueron cuatro grandes temáticas las que estructuraron el seminario: la gestión, uso y apropiación de los espacios públicos en la ciudad contemporánea; la gentrificación, renovación urbana y política urbana; el gobierno, políticas y planificación urbana; y las políticas de infraestructuras urbanas: planificación, gestión e impactos urbanos.

El evento ha sido un real éxito, en varios aspectos. Un éxito en términos de participación: primero por el interés que suscitó tanto para la presentación de trabajos académicos como para participar, superando todas las expectativas. Fueron más de 300 solicitudes de ponencias, de las cuales fueron seleccionadas unas 70.

2 Tal como lo propuso Jordi Borja en la conferencia inaugural: «El derecho a la ciudad y ciudadanía. El dilema entre los derechos sociales y los derechos políticos». 


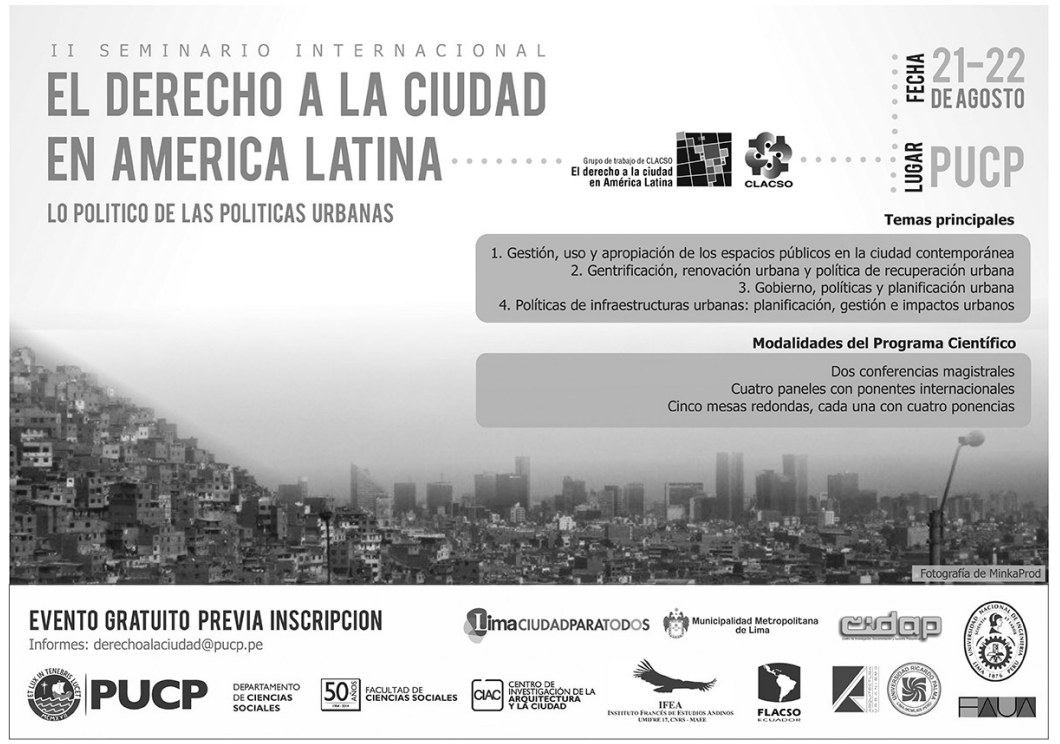

Las capacidades previstas también fueron rebasadas: los cupos de inscripciones se agotaron en dos días (hubieron más de 600 solicitudes de inscripción) y se acogieron a más de 200 personas el primer día (acondicionando dos aulas con retransmisión de video en vivo). Esto muestra el interés por esta temática y la necesidad de generar más espacios de este tipo, en particular en Perú.

Un éxito internacional: la mayor parte de los ponentes han venido del extranjero, sobretodo de los países de la región (México, Ecuador, Chile, Argentina, Colombia) pero también de Estados Unidos y Europa. La formación de redes de investigadores es uno de los objetivos del grupo del Derecho a la Ciudad de CLACSO, y este evento evidenció el potencial que existe en América Latina, la necesidad de fortalecer los lazos existentes así como los desafíos en particular para el país anfitrión de seguir apoyando a una investigación urbana todavía muy tímida.

Un éxito intergeneracional: la mezcla de niveles y generaciones era uno de los objetivos que también se concretó, con la presentación de tesis de licenciatura, maestría y de doctorandos al lado de grandes figuras como Jordi Borja (España), Carlos De Mattos (Chile), Fernando Carrión (Ecuador), Felipe Burbano (Ecuador), Joan Subirats (España), Cristina Cravino (Argentina), Alicia Ziccardi (México), Pablo Vega Centeno (Perú), Gustavo Riofrío (Perú), Alfredo Rodríguez (Chile) y René Coulomb (México), entre otros. El diálogo es enriquecedor, comprobando la actualidad del tema, sin negar la pertinencia de miradas más experimentadas capaces de tomar distancia.

En fin, un éxito temático y multidisciplinario: pudo reunir una gran cantidad de disciplinas de las ciencias sociales, desde la Sociología, Antropología, Arquitectura, Economía, Ciencias Políticas o Geografía, entre otros, alrededor de un eje de reflexión común. La riqueza de las intervenciones y los nuevos cuestionamientos 
levantados sobre lo político de las políticas urbanas demostraron la pertinencia de la temática central del seminario.

Tantos éxitos merecen una crítica, que no deja de ser constructiva para el dibujo de líneas para futuras investigaciones. Se evidenciaron dos dificultades principales: la primera concierne a la necesidad de ir más allá del caso de estudio para generalizar y crear conocimiento. Justifica la existencia de una red, que ofrece una plataforma para el desarrollo de estudios comparativos. Evidencia también la necesidad de espacios de discusión y reflexión para hacer emerger las estructuras detrás de los casos, y que deben acompañar este tipo de seminarios donde el tiempo queda corto para el debate.

La segunda dificultad es más bien un desafío y un llamado a renovar y reconstruir las preguntas de investigación. En este sentido, los mismos defensores del Derecho de la Ciudad no dejan de recordar que el uso del concepto esconde a veces un cierto «hacer lo mismo, pero cambiando las palabras».

Plantear el enfoque desde lo político era una manera de distanciarse de los discursos convencionales y, al afrontarse a los cambios contemporáneos, de innovar sobre una cuestión ya bastante recorrida. Cabe resaltar (entre otros) los trabajos presentados por Joan Subirats (Universidad Autónoma de Barcelona) que busca identificar las formas de respuestas de las ciudades españolas después de la crisis de 2007, o de Fernando Carrión y Felipe Burbano sobre el giro a la izquierda de las ciudades en América Latina. Estas investigaciones invitan a la exploración de un nuevo campo que abarca las dimensiones políticas de los problemas urbanos. Permite también ir más allá de la crítica al neoliberalismo (a veces demasiado fácil, en particular por la falta de opositores), para interrogar las nuevas formas de gobernabilidad y los desafíos políticos a los cuales se enfrentan nuestras ciudades. Ponen en alto la necesidad siempre vigente de construir y reconstruir la producción científica sobre la ciudad.

\section{Referencia citada}

FIGARI GOLD, E. \& RICOU, X., 1991 - Lima en crisis: Propuestas para la gestión de los servicios urbanos en Lima Metropolitana, 160 pp.; Lima: Instituto Francés de Estudios Andinos, Universidad del Pacífico.

Jérémy ROBERT, Cyriaque HATTEMER 